\title{
Bidirectional Nonreciprocal Wavelength-Interleaving Coherent Fiber Transversal Filter
}

\author{
Yicheng Lai, W. Zhang, J. A. R Williams, Member, IEEE, and I. Bennion, Member, IEEE
}

\begin{abstract}
A bidirectional nonreciprocal wavelength-interleaving filter based on an optically coherent high birefringence fiber transversal filter structure is demonstrated. Stable, low loss, low dispersion, and high isolation operation is demonstrated with reconfigurable transfer characteristics for interleaved channel spacing of $0.8 \mathrm{~nm}$.
\end{abstract}

Index Terms-Bidirectional transmission, optical fiber delay lines, optical fiber filters, wavelength-division multiplexing (WDM).

\section{INTRODUCTION}

$\mathbf{I}$ $\mathrm{N}$ A high-capacity wavelength-division-multiplexed (WDM) communication system, the use of nonreciprocal wavelength-interleaved bidirectional transmission over a single fiber reduces the use of fiber infrastructure by a factor of two. Besides alleviating nonlinear interactions between adjacent channels, the transmission technique suppresses optical backreflections to prevent signal degradations and oscillations in amplifiers [1], [2]. This wavelength allocation and isolation scheme for the opposing transmitted wavelength channels (denoted as odd and even channels) requires an enabling optical device that exhibits complementary periodic wavelength transfer functions between opposing transmission ports. Desirable device features for a high level of performance include low loss, low dispersion, good isolation, and reconfigurable transfer responses. Implementations involving optical path separations through circulators, multiplexer-demultiplexer modules, and filters have been demonstrated, e.g., [2]. However, such schemes compromise its noise figure performance [3] and are restricted by the operation bandwidth of the components. Alternative approaches using unique birefringent crystal and waveplate-based configurations have also been proposed, e.g., [3], [4].

The coherent optical delay line/transversal filter is an attractive technology for signal processing in optical communication systems [5]. Its operation enables a variety of complex frequency transfer functions like high-pass filtering and flattop passbands without inherent power loss. A dispersionless coherent transversal filter on a silica-based waveguide has recently been demonstrated [6]. By exploiting the intrinsic superior propagation properties of optical fibers, coherent fiber-based transversal filters further achieve high-performance ultrabroad-band signal processing with merits like engineering simplicity, fiber compatibility, low loss, and low dispersion.

Manuscript received July 18, 2003; revised October 9, 2003.

The authors are with Photonics Research Group, Aston University, Birmingham B4 7ET, U.K. (e-mail: laiy@ aston.ac.uk).

Digital Object Identifier 10.1109/LPT.2003.821242

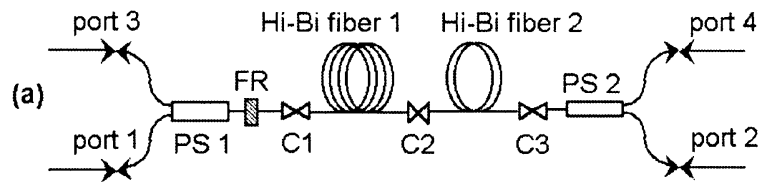

(b)

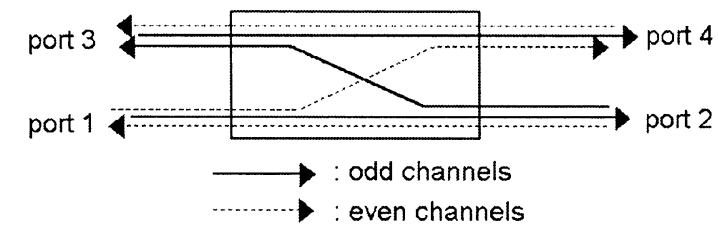

Fig. 1. (a) Hi-Bi fiber transversal filter configuration. (b) The equivalent block diagram. C1-4: connector point.

We had previously reported a coherent fiber transversal filter that overcomes environmental sensitivity typical of coherent fiber devices by utilizing noninterfering orthogonal polarized modes in a high birefringence (Hi-Bi) fiber [7]. Instead of using separate optical paths, the scheme utilizes the birefringence in the Hi-Bi fiber to achieve the desired optical path difference (OPD). Optical taps within the fiber experience common mode suppression of ambient perturbations for stable long-term operation without stringent environmental isolation. In this letter, we demonstrate a new approach to realize a bidirectional, nonreciprocal wavelength-interleaving filter device based on such a coherent Hi-Bi fiber delay line structure. Without expensive birefringent crystal-based inner stages, the architecture achieves low loss performance and ease of implementation. The filter features reconfigurable wavelength transfer function, wide operation bandwidth, and negligible chromatic dispersion. Bidirectional, nonreciprocal, wavelength-interleaving notch, and flattop bandpass filtering are demonstrated for interchannel spacing of $0.8 \mathrm{~nm}$ with $>26-\mathrm{dB}$ isolation.

\section{BACKGROUND}

The configuration of the filter structure is shown in Fig. 1(a). The wavelength-selective transversal filter element is formed by Hi-Bi Fiber 1 and 2 of length ratio $2: 1$. Polarization splitters PS 1 and PS 2 couple light to and from its input-output ports onto the propagation axes of the Faraday rotator (FR) and Hi-Bi Fiber 2 , respectively. The off-the-shelf fiber pigtailed FR creates a $90^{\circ}$ polarization plane rotation on light that propagates in reverse direction (Port $2 \rightarrow$ Port 1 ).

Depicted in Fig. 2(a), in the forward operation (Port $1 \rightarrow$ Port 2), the Port 1 input light (after the FR) launched into Hi-Bi Fiber 1 at an azimuth $\theta_{1}$ degenerates into two orthogonal polarized modes $x, y$. These optical taps with a delay interval $\tau_{1}$ given by $\tau_{1}=\Delta n \cdot L_{1} / c$, where $c$ is the speed of light, $L_{1}$ is the length of Hi-Bi 1, and $\Delta n$ is the fiber birefringence, couples into Hi-Bi 
(1) $\mathrm{Hi}-\mathrm{Bi}$ fiber 1

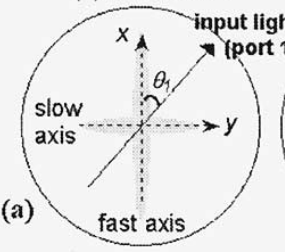

(2) Hi-Bi fiber 2

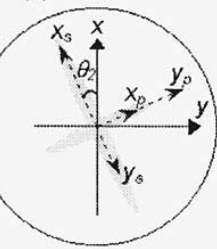

(3) Polarization splitter 2

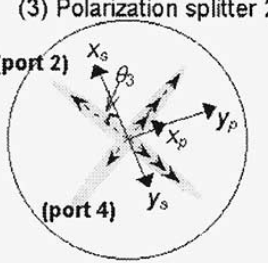

(1) Hi-Bi fiber 2

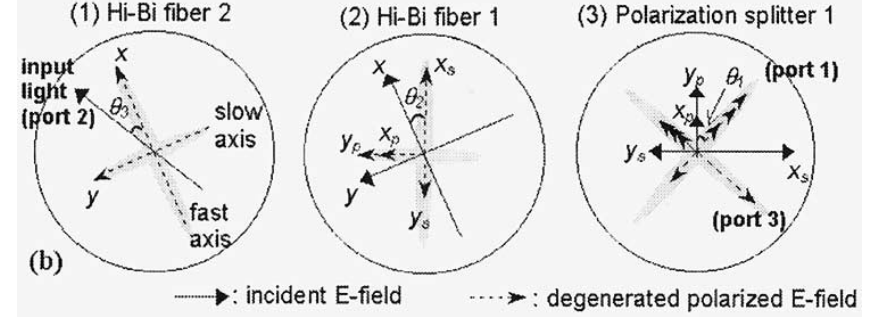

Fig. 2. (a) Figures depicting degeneration of $E$-fields in the forward operation. (b) Figures depicting degeneration of $E$-fields in the reverse operation.

Fiber 2 at an azimuth $\theta_{2}$ and degenerate to form polarized modes $x_{s}, x_{p}, y_{s}, y_{p}$, sequentially. Delay interval $\tau_{2}$ between these temporally evenly spaced signal taps is given by $0.5 \tau_{1}$ due to the $\mathrm{Hi}$-Bi fiber length ratio. Subsequently, these polarized modes couple into the propagation axes of PS 2 to form the signal taps that determine the wavelength transfer characteristics at output Ports 2 and 4.

In the reverse operation, as depicted in Fig. 2(b), similar polarization mode degeneration occurs as the input light propagates from Port 2 toward Port 1 . The only difference lies in the $90^{\circ}$ polarization plane rotation (with respect to propagation axes of PS 1) by the FR before the optical taps $x_{s}, y_{s}, x_{p}, y_{p}$ couple from Hi-Bi 1 into the propagation axes of PS 1 . Note that the degenerated polarized modes $x, y$ from the Port 2 input light are temporally spaced at $\tau_{2}$ and the delay interval between subsequent degenerated modes $x_{s}, x_{p}$ or $y_{s}, y_{p}$ in Hi-Bi 1 is given by $2 \tau_{2}$ as a result of the Hi-Bi fiber length ratio. Consequently, the temporal sequence, in increasing order of time, of the output signal taps is $x_{s}, y_{s}, x_{p}, y_{p}$ with delay interval similarly given by $0.5 \tau_{1}$.

It is evident that the electric field amplitudes of all the polarized modes (hence, tap coefficients) are determined by coupling angles $\theta_{1}, \theta_{2}$, and $\theta_{3}$ only. Unlike optical transversal filters using silica-based waveguides, determination of the tap weightings can be computed at ease without impulse response measurement. Furthermore, with commercially available $\mathrm{Hi}-\mathrm{Bi}$ fiber keyed connectors and precision rotators, angles $\theta_{1}, \theta_{2}$, and $\theta_{3}$ can be, in practice, set to an accuracy of $<0.1^{0}$. Based on Fig. 2, the electric field amplitudes (tap coefficients) at output Port 2 in the forward operation and output Port 1 in the reverse operation can be expressed as

$$
\left[\begin{array}{c}
-\cos \theta_{1} \cdot \cos \theta_{2} \cdot \cos \theta_{3} \\
\cos \theta_{1} \cdot \sin \theta_{2} \cdot \sin \theta_{3} \\
\sin \theta_{1} \cdot \sin \theta_{2} \cdot \cos \theta_{3} \\
\sin \theta_{1} \cdot \cos \theta_{2} \cdot \sin \theta_{3}
\end{array}\right] \text { and }\left[\begin{array}{c}
\sin \theta_{1} \cdot \cos \theta_{2} \cdot \cos \theta_{3} \\
-\sin \theta_{1} \cdot \sin \theta_{2} \cdot \sin \theta_{3} \\
\cos \theta_{1} \cdot \sin \theta_{2} \cdot \cos \theta_{3} \\
\cos \theta_{1} \cdot \cos \theta_{2} \cdot \sin \theta_{3}
\end{array}\right]
$$

respectively. Due to the degeneration of the electric fields propagating through the cascaded structure, there is one tap negated with respect to the rest in each output group and at a different

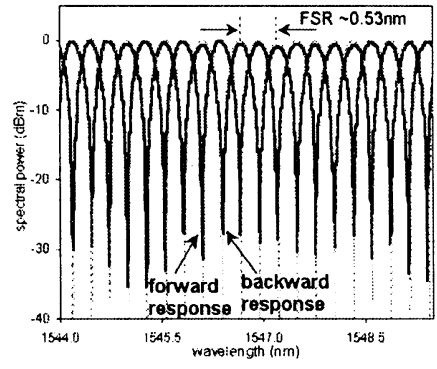

(a)

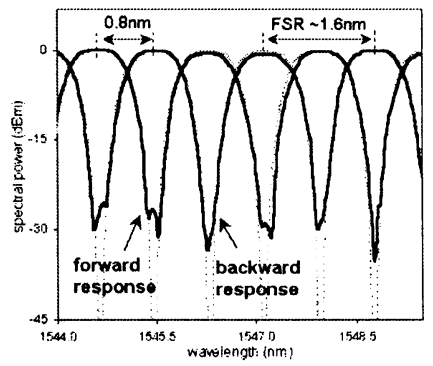

(c)

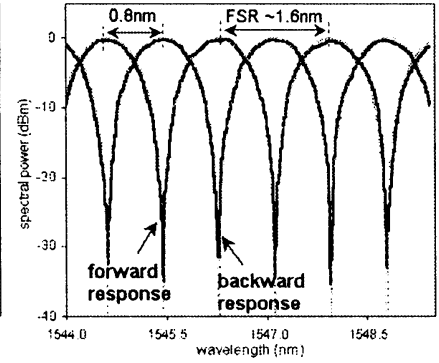

(b)

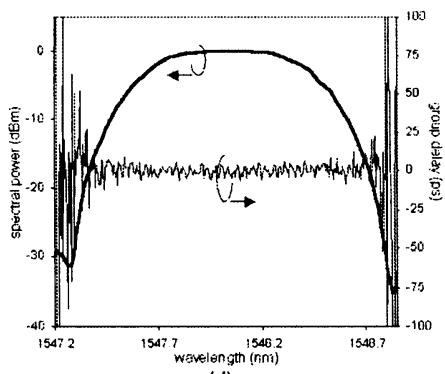

(d)
Fig. 3. Simulation (dotted) and measured forward and backward responses of the filter at Ports 1 and 2 when (a) $\theta_{1}=45^{\circ}, \theta_{2}=0^{\circ}$, and $\theta_{3}=45^{\circ}$; (b) $\theta_{1}=45^{\circ}, \theta_{2}=90^{\circ}$, and $\theta_{3}=45^{\circ}$; and (c) $\theta_{1}=75^{\circ}, \theta_{2}=58^{\circ}$, and $\theta_{3}=45^{\circ}$. (d) Group delay response when $\theta_{1}=75^{\circ} ; \theta_{2}=58^{\circ} ; \theta_{3}=45^{\circ}$.

order of occurrence. Nonreciprocal response is achieved between the output ports as a result of this difference in the order of occurrence of the negated tap between the counterpropagating operations. The measured optical response of the filter is the magnitude of the coherent sum of the constituent electric fields. Since the tap coefficient is a function of the coupling angles, a variety of wavelength transfer characteristics can be achieved by simply varying $\theta_{1}, \theta_{2}$, and $\theta_{3}$. The free spectral range (FSR) of the output response, determined by the tap interval, is given by $1 /\left(0.5 \tau_{1}\right)$. Accurate alignment to the desired FSR can be achieved by suitable choice of Hi-Bi fiber length and birefringence. The above analysis extends similarly to Port $3 \leftrightarrow$ Port 4 , Port $3 \leftrightarrow$ Port 2 , and Port $1 \leftrightarrow$ Port 4 . Note that complementary responses are obtained between Ports 1 and 3 and Ports 4 and 2 [7]. The operation of the filter is depicted in Fig. 1(b) by the propagation paths of the odd and even channels.

\section{EXPERIMENT}

Two pieces of Hi-Bi fiber with lengths 4.50 and $2.25 \mathrm{~m}$, respectively, were used in the experiment. The beat length of the $\mathrm{Hi}$-Bi fiber was $2.3 \mathrm{~mm}$ corresponding to a birefringence of $6.7 \times 10^{-4}$. The cleaved ends of the fibers are butted together (at $\mathrm{C} 1, \mathrm{C} 2$, and $\mathrm{C} 3$ ) such that by rotating the fiber ends with respect to each other, the orientation of the input light with respect to the birefringence axes can be varied. No special attention was required to isolate the setup from environmental perturbations. Under two special cases $\left(\theta_{1}=45^{\circ}, \theta_{2}=0^{\circ}, \theta_{3}=45^{\circ}\right.$ and $\left.\theta_{1}=45^{\circ}, \theta_{2}=90^{\circ}, \theta_{3}=45^{\circ}\right)$, the relative orientation between the birefringence axes of Hi-Bi Fiber 1 and 2 are aligned and orthogonal, respectively. This reconfigures the structure to a two-tap optical notch filter with maximum tap time interval for the former and minimum tap time interval for the latter. Fig. 3 shows the superimposed measured forward and reverse filter responses between Ports 1 and 2 at $\theta_{1}=45^{\circ}, \theta_{2}=0^{\circ}$, and 
$\theta_{3}=45^{\circ}$ [Fig. 3(a)]; and $\theta_{1}=45^{\circ}, \theta_{2}=90^{\circ}$, and $\theta_{3}=45^{\circ}$ [Fig. 3(b)]. The obtained spectrum agrees well with the simulation results (dotted traces). The measured FSR are 0.53 and $1.6 \mathrm{~nm}$, respectively, with notch depth measured to be $>30 \mathrm{~dB}$. Nonreciprocal and wavelength-interleaving filter characteristics are clearly evident. Furthermore, by setting $\theta_{1}=75^{\circ}, \theta_{2}=58^{\circ}$, and $\theta_{3}=45^{\circ}$, a flattop bandpass response, as shown in Fig. 3(c), is realized. This response is a characteristic feature of a coherent transversal filter consisting of bipolar optical taps. The flattened passband width measured $0.32 \mathrm{~nm}$ with ripples $<0.1 \mathrm{~dB}$. Sidelobe suppression $>26 \mathrm{~dB}$ is obtained and the FSR is $1.6 \mathrm{~nm}$, corresponding to interleaved channel spacing of $0.8 \mathrm{~nm}$. The measured ripple can be attributed to the fluctuation in the measurement laser. Slight Hi-Bi fiber length ratio and angular discrepancies account for the less than ideal sidelobe suppressions. The length ratio mismatch can be thermally compensated over a small isolated section $(10 \mathrm{~cm}$ ) of the Hi-Bi fiber (without coating) and the birefringence variation with temperature measured $\sim-2 \times 10^{-7} /{ }^{\circ} \mathrm{C}$. It is worth noting that any instability in the device is associated with OPD variations when the fiber birefringence is disturbed. Commercially available $\mathrm{Hi}-\mathrm{Bi}$ fibers generally have thick $400-\mu \mathrm{m}$ coating that reduces significantly the sensitivity to environmental perturbations [8]. If thick nylon coating is used, the temperature sensitivity can be further reduced by $95 \%$ [8]. In the experiment, under normal laboratory environment, the stability of the device is exemplified by the long measurement time of the transfer responses.

The group delay characteristic of the filter across a wavelength channel when $\theta_{1}=75^{\circ}, \theta_{2}=58^{\circ}$, and $\theta_{3}=45^{\circ}$ was measured using the modulation phase shift technique. As shown in Fig. 3(d), negligible chromatic dispersion is evident by the fact the peak-to-peak group delay ripple across the passband of the filter response is $< \pm 6$ ps at modulation frequency of $200 \mathrm{MHz}$. The system noise contribution during measurement was found to be in the same order of magnitude. The ultralow dispersion characteristics extend to all wavelength channels. Random group delay noise values outside the passband of the filter response are attributed to the low transmitted optical power.

Contrary to [2]-[4], excess loss within the device is not attributed to the wavelength-selective filter element but due to the insertion loss of the polarization rotator and splitters measured
$<1 \mathrm{~dB}$. Since the concept of the filter applies to a greater number of Hi-Bi fiber sections [7], enhanced filter response with higher sidelobe suppression due to the increased number of optical taps can, hence, be realized without compromising insertion loss. Since the material dispersion of the Hi-Bi fiber is very small, the wavelength transfer characteristics of the filter extend without distortion across the entire $C$-band. More importantly, bidirectional amplification can be further achieved by simply using Hi-Bi Erbium-doped fibers. Contrary to all reported schemes, the absence of additional cascaded unidirectional amplifiers or circulars will enable high-performance low noise figure bidirectional amplification. Further results will be presented in the near future.

In summary, a bidirectional, nonreciprocal, wavelength-interleaving filter for WDM systems is demonstrated. The device features various advantages and can be easily adapted to perform bidirectional amplification without additional components. Results obtained highlight its potential applications in advanced optical communication systems.

\section{REFERENCES}

[1] J. Kani, M. Jinno, T. Sakamoto, K. Hattori, and K. Oguchi, "Bidirectional transmission to suppress interwavelength-band nonlinear interactions in ultrawide-band WDM transmission systems," IEEE Photon. Technol. Lett., vol. 11, pp. 376-378, Mar. 1999.

[2] S. K. Liaw, K. P. Ho, C. Lin, and S. Chi, "Multichannel bidirectional transmission using a WDM MUX/DMUX pair and unidirectional in-line amplifiers," IEEE Photon. Technol. Lett., vol. 9, pp. 1664-1666, Dec. 1997.

[3] T. W. Oh et al., "Bidirectional erbium-doped fiber amplifier with nonreciprocal optical filter," Electron. Lett., vol. 37, no. 5, pp. 283-284, 2001.

[4] T. Ducellier et al., "Bidirectional circulator: An enabling technology for wavelength interleaved bidirectional networks," in Proc. 26th Eur. Conf. Optical Communication (ECOC) 2000, Munich, 2000, Postdeadline paper PD3-9.

[5] K. P. Jackson et al., "Optical fiber delay-line signal processing," IEEE Trans. Microwave Theory Tech., vol. MTT-33, pp. 193-210, Mar. 1985.

[6] T. Mizuno et al., "Dispersionless interleave filter based on transversal form optical filter," Electron. Lett., vol. 38, no. 19, pp. 1121-1122, 2002.

[7] Y. Lai, W. Zhang, J. A. R. Williams, and I. Bennnion, "A novel dual complementary output optical fiber transversal filter for DWDM applications," in Proc. 15th Annu. Meeting LEOS 2002, Glasglow, U.K., 2002, Paper ThE 1.

[8] J. Noda, K. Okamoto, and Y. Sasaki, "Polarization maintaining fibers and their applications," J. Lightwave Technol., vol. LT-4, pp. 1071-1089, Aug. 1986. 